\title{
Loslas profesionales de la salud frente a la Ley de Identidad de Género argentina. Tensiones entre el saber experto y el cuidado integral
}

\section{| ${ }^{1}$ Anahí Farji Neer I}

Resumen: El artículo analiza la recepción de la Ley de Identidad de Género por parte de profesionales de la salud del Área Metropolitana de Buenos Aires en base al análisis de un corpus de entrevistas en profundidad a profesionales de las especialidades de Salud Mental, Endocrinología y Cirugía que realizan o evalúan el ingreso a tratamientos hormonales y/o quirúrgicos solicitados por mujeres y varones trans. La Ley 26.743/12 de Identidad de Género argentina legaliza las intervenciones médicas para que travestis, transexuales, transgéneros y trans adquieran una imagen corporal acorde a su identidad de género. Dispone la cobertura de los tratamientos por parte del sistema de salud público, obras sociales y prestadores privados. A diferencia del régimen legal vigente con anterioridad, no exige requisitos diagnósticos ni judiciales para acceder a dichos tratamientos. El artículo describe los criterios adoptados tras la aprobación de la Ley de Identidad de Género por equipos y profesionales para evaluar el ingreso y resultados de dichos tratamientos. Las principales conclusiones establecen que la Ley de Identidad de Género habilitó tres desplazamientos en los discursos vigentes en el campo médico local: del diagnóstico al acompañamiento, del protocolo a la personalización-customización y de la minimización del riesgo al cálculo costo-beneficio.

> Palabras clave: derecho a la identidad de género; servicios de salud para personas trans; despatologización; medicalización.
${ }^{1}$ Instituto de Investigaciones Gino Germani, Universidad de Buenos Aires. Buenos Aires, Argentina (anahifarji@hotmail.com). ORCID: 0000-0002-4953-7672
Recebido em: 10/08/2017 Revisado em: 11/06/2018 Aprovado em: 22/06/2018 


\section{Introducción}

En 2012, en Argentina fue aprobada la Ley 26.743 de Identidad de Género que permite a cualquier persona cambiar de nombre y sexo en su documento de identidad mediante la realización de un trámite personal ante las oficinas del registro civil. Para menores de edad, la Ley establece que el trámite sea realizado por intermedio de sus representantes legales, debiendo contar además con el patrocinio de un abogado del niño ${ }^{1}$. Para mayores de edad, dispone que quienes así lo deseen puedan acceder a prácticas médicas para construir una corporalidad acorde a su identidad de género, estableciendo como único requisito la firma de un consentimiento informado. Para que menores de edad puedan acceder a dichas prácticas médicas, la Ley establece los mismos requisitos que para el cambio de nombre y sexo registral. No obstante, establece que niños, niñas y adolescentes que deseen acceder a intervenciones quirúrgicas, deberán contar con una autorización judicial para poder realizarlas. El decreto de reglamentación contempla a las personas migrantes cuyos países de origen no poseen normativas que les permitan realizar los cambios registrales requeridos. De este modo, aquellas personas migrantes con residencia permanente en el país cuyos países de origen no cuenten con normativas que contemplen su derecho a la identidad de género, podrán realizar las modificaciones registrales en el documento nacional de identidad para extranjeros/as ${ }^{2}$. El contenido de esta legislación fue elaborado por las organizaciones de travestis, transexuales, transgéneros y trans argentinas, las que demandaron la eliminación de los requisitos diagnósticos y judiciales anteriormente vigentes para acceder a estos derechos.

El presente trabajo aborda la recepción de la Ley de Identidad de Género entre los/ as profesionales de la salud del Área Metropolitana de Buenos Aires especializados/ as en tratamientos de construcción corporal en mujeres y varones trans. Analiza los cambios y continuidades en sus discursos y prácticas respecto a los criterios vigentes en el campo médico global con anterioridad a la aprobación de la Ley de Identidad de Género. Describe los enfoques desarrollados por los/as distintos/as profesionales y equipos respecto a los criterios de ingreso, indicación de tratamiento y evaluación de resultados. Para ello se desarrolló un diseño cualitativo basado en el análisis de entrevistas en profundidad a profesionales de las especialidades de Salud Mental, Endocrinología y Cirugía del Área Metropolitana de Buenos Aires, que entre los años 2014 y 2016 realizaban o evaluaban el ingreso a tratamientos hormonales y/o 
quirúrgicos en mujeres y varones trans. Se realizó un total de ocho entrevistas. Se entrevistaron dos profesionales de Salud Mental, tres cirujanos/as y tres endocrinólogos/ as que se desempeñaban en la Ciudad de Buenos Aires, Ciudad de La Plata y Partido de San Martín de la Provincia de Buenos Aires. En las entrevistas se indagó en su trayectoria profesional, en los cambios en la organización de la atención dispuestos a partir de la sanción de la Ley de Identidad de Género y en sus percepciones sobre el paradigma despatologizante plasmado en el contenido de la Ley. Cada entrevista tuvo una duración aproximada de 45 minutos y fue realizada tras la firma del formulario de consentimiento informado supervisado y aprobado por el Comité de Ética del Instituto de Investigaciones Gino Germani de la Universidad de Buenos Aires. Las entrevistas fueron desgrabadas, cargadas en un software para el análisis de datos cualitativos y codificadas en base a las dimensiones de análisis indicadas. El contenido del presente artículo forma parte de la tesis doctoral de la autora, desarrollada en el marco del programa de Doctorado en Ciencias Sociales de la Facultad de Ciencias Sociales de la Universidad de Buenos Aires (FARJI NEER, 2016a) y también forma parte del informe final de la Beca Carrillo-Ońativia otorgada por el Ministerio de Salud de la Nación para el período 2015-2016 (FARJI NEER, 2016b).

\section{Del consultorio a las calles: nacimiento y transformación de un diagnóstico}

El nacimiento de la transexualidad como categoría médica e identitaria, asociada a distintos procesos de construcción corporal medicalizados, puede ser leído como resultado del avance del conocimiento endocrino y perfeccionamiento de las técnicas quirúrgicas (HAUSMAN, 1995). El término “Transexual psíquico” fue acuñado en 1923 por el médico alemán Magnus Hirschfeld, quien desarrolló la teoría de los "estadios sexuales intermedios". El término fue retomado en 1949 por el médico estadounidense David Cauldwell. En su texto Psychopathia Transexualis, lo describió como una predisposición orgánica hereditaria que, combinada con una crianza disfuncional, podría producir una variedad de efectos psicológicos entre los cuales se encontraría la creencia de pertenecer al otro sexo. Lo entendió como un cuadro que podría mejorar o incluso alcanzar la cura a través del tratamiento psicológico (CAULDWELL, 2006), idea que fue retomada luego por el psicoanálisis lacaniano (BARBOSA, 2015). 
A fines de la década de 1960 Harry Benjamin, endocrinólogo alemán radicado en Estados Unidos, estableció las bases para diagnosticar el "verdadero transexualismo". A diferencia de los abordajes psicoanalíticos, desde su perspectiva las intervenciones quirúrgicas y hormonales podían constituir la terapéutica indicada para los cuadros de transexualismo, siempre que se corroborara previamente la autenticidad de dicho cuadro patológico. Estos desarrollos se plasmaron en una serie de protocolos aplicados a mediados de 1960 en las Clínicas de Identidad de Género creadas en Estados Unidos y replicadas luego en diversas partes del mundo. En 1979 Benjamin editó los Standards of Care for Gender Identity Disorders (en adelante SOC) en los que estableció un método estandarizado para el diagnóstico y tratamiento del transexualismo. Inicialmente el proceso de diagnóstico se orientaba a corroborar tres aspectos: el sentimiento de pertenecer al sexo opuesto, el uso temprano y persistente de vestimentas del sexo opuesto sin un sentido erótico y el desprecio hacia el comportamiento sexual homosexual. Pasado el proceso de diagnóstico, el tratamiento se componía de tres etapas progresivas e inescindibles: una psicológica, una hormonal y por último, una quirúrgica.

En la década de 1980, el “Transexualismo" primero y el "Trastorno de la identidad de género" luego fueron incluidos en los manuales de clasificación de enfermedades y trastornos mentales de circulación mundial: el Manual Diagnóstico y Estadístico de los Trastornos Mentales (DSM) de la Asociación Americana de Psiquiatría y la Clasificación Internacional de Enfermedades (CIE) de la Organización Mundial de la Salud ${ }^{3}$. Tras la muerte de Benjamin en 1986, los SOC pasaron a ser revisados por la World Professional Association for Transgender Health (WPATH) que, al igual que los manuales diagnósticos, son revisados periódicamente. En su $7^{\circ}$ versión del año 2011, los SOC introdujeron conceptos que reconocen las múltiples posibilidades identitarias y corporales, en sintonía con el reclamo de despatologización de las identidades trans sostenido por el activismo trans a nivel global (COLL-PLANAS, 2010; SUESS, 2010). Dicho documento afirma que la "Variabilidad de género" o "No conformidad de género" y la "Disforia de género" no son fenómenos necesariamente vinculados. Por "No conformidad de género" o "Variabilidad de género" entiende aquellas formas de identificación o expresión de género que difieren de las normas culturales establecidas. "Disforia de género" es el término utilizado para referir a la "incomodidad o malestar causado por la discrepancia entre la identidad de género y el sexo asignado a la persona al nacer (y el papel de género asociado y/o las 
características sexuales primarias y secundarias)" (WPATH, 2011, p. 5). En sintonía con estas transformaciones, el DSM 5 publicado en 2013 destinó un capítulo específico a la categoría "Disforia de género", a la que escindió de las "Disfunciones sexuales y parafilias". Allí incorporó la noción de "No conformidad de género" en un sentido que no refiere necesariamente a una experiencia de sufrimiento psíquico.

Hasta 2012, en Argentina los pedidos de cambio registral en los documentos identificatorios y de realización de cirugías genitales para construir una corporalidad acorde a la identidad de género requerían de una autorización judicial, según lo establecido en el Código Penal, la Ley 17.132 de Ejercicio de la Medicina y la Ley 18.248 del nombre. Los procesos judiciales imponían la certificación del diagnóstico psiquiátrico de "Trastorno de la identidad de género" (CABRAL, 2007; FARJI NEER, 2013; 2016). Las organizaciones de travestis, transexuales, transgéneros y trans argentinas conformadas a mediados de la década de 1990 denunciaron estos procedimientos como invasivos y violatorios del derecho a la intimidad y de los derechos sexuales, en definitiva, de los derechos humanos de los y las trans. A su vez, informes elaborados por sus organizaciones arrojaron que con anterioridad a la sanción de la Ley de Identidad de Género los y las trans evitaban acudir a la consultas sanitarias debido a las burlas y maltratos sufridos por parte de profesionales y personal administrativo (BERKINS; FERNÁNDEZ, 2004; BERKINS, 2007; FRIEDER; ROMERO, 2014). Este hecho se sumaba a las barreras burocráticas ocasionadas por el hecho de no contar con documentación que reflejase su identidad autopercibida. Todo ello desincentivaba y obstaculizaba el acceso a la atención sanitaria y propiciaba el ingreso a un circuito precario y clandestino de implantes, cirugías estéticas y prácticas de riesgo, tales como la inyección silicona líquida y el consumo de hormonas sintéticas sin supervisión médica (BERKINS, 2007; BERKINS; FERNÁNDEZ, 2004; FRIEDER; ROMERO, 2014).

El artículo $11^{\circ}$ de la Ley de Identidad de Género titulado "Derecho al libre desarrollo personal" establece que toda persona mayor de edad que así lo desee puede acceder gratuitamente a tratamientos médicos destinados a adecuar la corporalidad a la identidad de género, estableciendo como único requisito la firma de un consentimiento informado. El artículo también establece que menores de edad deben contar con la autorización de sus representantes legales y en caso de solicitar intervenciones quirúrgicas, una autorización judicial. Amparándose en el concepto de salud integral, incorpora dichas prestaciones al Plan Médico Obligatorio. Es 
decir, establece su cobertura por parte los tres subsistemas de salud (público, privado y obras sociales). Esta normativa dio encuadre legal a prácticas médicas que ya se venían realizando de modo informal o bien supeditado a una autorización judicial. Promovió la incorporación de nuevas especialidades y profesionales a los equipos interdisciplinarios que originalmente se componían de las especialidades de Cirugía Urológica, Endocrinología y Salud Mental, así como incentivó la conformación de nuevos equipos en instituciones de salud a lo largo del país.

La Ley de Identidad de Género propició las condiciones sociales y legales para que los discursos expertos de la medicina se vieran interpelados por las demandas de los y las trans, quienes de modo organizado o a título individual reclaman mayor participación en la toma de decisiones sobre el curso de los tratamientos y tensionan las formas instituidas de la autoridad médica y de la relación médico/ a-paciente. Con el fin de indagar en los cambios y continuidades respecto a los criterios médicos vigentes tras la sanción de la Ley de identidad de Género, a continuación se describen los enfoques sostenidos por profesionales de la salud que entre 2014 y 2016 realizaban o evaluaban el ingreso a los tratamientos hormonales y quirúrgicos de construcción corporal trans en el Área Metropolitana de Buenos Aires. Se analizan sus definiciones de los criterios de ingreso, indicación de tratamiento y evaluación de resultados adoptados o mantenidos tras la aprobación de la Ley. Se identifican distintos desplazamientos discursivos que dan cuenta de una reapropiación local de las transformaciones en los manuales diagnósticos y guías de atención de circulación global.

\section{¿Una autoridad médica reconfigurada?}

\section{Del diagnóstico al acompañamiento}

Entre 2014 y 2016, la mayoría de los/as profesionales especializados en salud trans atendía de modo interdisciplinario tanto en centros públicos como en consultorios privados y de obras sociales. Algunos equipos preexistían a la Ley de Identidad de Género y otros se conformaron con posterioridad a su sanción, ya sea por iniciativa de sus profesionales o bien de las direcciones médicas de sus instituciones. Eran equipos coordinados de modo centralizado por un/a profesional que pertenecía a la especialidad de Psiquiatría, Cirugía o Endocrinología. Este/a profesional era el/la encargado/a de organizar el trabajo entre las distintas especialidades que integraban 
los equipos interdisciplinarios y realizaba las entrevistas de admisión, evaluación y derivación de los/as pacientes. Ni la Ley o su reglamentación, ni el Ministerio de Salud Nacional a través de ordenanzas u otros instrumentos regulatorios, establecieron taxativamente un modelo de atención. Cada equipo tenía entonces la potestad de elaborar sus propias guías de atención basadas en las guías locales e internacionales, la experiencia clínica acumulada y/o las concepciones y criterios de sus integrantes.

Una de las principales directrices que comenzó a estructurar la atención, en algunos casos con anterioridad a la sanción de la Ley de Identidad de Género, fue la evaluación integral (física y psicológica) de los/as pacientes, a fin de determinar en qué medida se encontraban preparados/as para iniciar los tratamientos solicitados, en especial los quirúrgicos. Los criterios que anteriormente tenían como fin corroborar o descartar el diagnóstico de "Transexualismo" o "Trastorno de la identidad de género", comenzaron a traducirse al lenguaje de la integralidad: los/as profesionales consideraban que era necesaria una evaluación integral para conocer las condiciones físicas, emocionales y psicológicas de los/as potenciales pacientes. Al decir de los/as profesionales, la evaluación poseía varios objetivos. En principio, entender o ayudar a construir la configuración identitaria del/la paciente y, a partir de allí, ofrecer las distintas opciones disponibles para la construcción corporal, informando sus potencialidades y riesgos. También ofrecer herramientas para tramitar la transición corporal en sus círculos sociales:

Lo que hacemos es detectar la necesidad de cada persona y a partir de su necesidad acompańamos, pero primero aclarar la situación de momento vital [...] que la persona tenga claro quién es y qué necesita, y a partir de eso acompańamos el tipo de tratamiento: hormonal, quirúrgico, total, parcial... eso se ve en cada caso en particular (J., psiquiatra).

Siguiendo a los/as profesionales entrevistados/as, las entrevistas de evaluación se orientaban a corroborar que la persona se encontraba emocionalmente apta para realizar los tratamientos, a fin de minimizar los riesgos de arrepentimiento. Para ello construían indicadores, contextualmente elaborados, de la estabilidad o inestabilidad en la toma de decisiones a lo largo de su vida. Un endocrinólogo describía el método adoptado del siguiente modo:

Hablando con él y preguntándole primero su historia, su acompañamiento familiar, su acompañamiento social, su inserción laboral, su aceptación en el entorno, viendo el aspecto que tiene, si tiene aspecto de hombre o de mujer, cómo se viste y preguntándole cómo son sus expectativas, si hizo algún otro tratamiento y tratando de ver un poco... Hay ciertos patrones que te pueden hablar de la persona. Por ejemplo, una persona que 
te puede llegar a decir "empecé abogacía y dejé, empecé arquitectura y dejé, empecé medicina y dejé, me gusta el teatro pero no soy constante", entonces vos podés inferir que por ahí no va a ser constante en su tratamiento (L., endocrinólogo).

La evaluación se basaba en las percepciones de los/as profesionales respecto a la trayectoria de vida de las personas solicitantes de los tratamientos, instituyendo un protocolo contextual e informal de evaluación de la propensión al arrepentimiento.

Luego de la primera entrevista, el tratamiento psicoterapéutico podía ser obligatorio u opcional, dependiendo del criterio utilizado por el equipo o el/ la profesional. Cuando se demandaba algún tipo de intervención quirúrgica, la interconsulta psicoterapéutica generalmente era obligatoria. Si la persona ya se encontraba realizando un tratamiento psicoterapéutico por su cuenta, los/as profesionales no indicaban el cambio de profesional, sino que solicitaban contactarse con este/a fin de realizar una evaluación conjunta. En este sentido, uno de los profesionales entrevistados refería:

Si ya vienen con una evaluación psiquiátrica de años y todas esas cosas y yo noto que está bien llevado, me parece agresivo cambiarle de terapeuta al final del camino. Entonces charlo con el terapeuta que lo está llevando y si me genera dudas, sí, lo hago evaluar por el equipo que yo trabajo. Si viene el paciente a la deriva y no tiene ningún tipo de evaluación lo enfoco y lo pongo en el sistema nuestro de evaluación (R., cirujano).

Pese a los reclamos del activismo local e internacional por la despatologización de las identidades trans (COLL-PLANAS, 2010; SUESS, 2010), entre 2014 y 2016 en varios servicios del Área Metropolitana de Buenos Aires el requisito del diagnóstico o autorización por parte de un/a profesional de Salud Mental continuaba siendo obligatorio para acceder a intervenciones quirúrgicas genitales. Los/as profesionales sostenían la necesidad de contar con dicho aval apelando a la irreversibilidad de las intervenciones y utilizando un discurso que se ubicaba a medio camino entre el cuidado y la tutela:

Hay algo que yo no cambio: lo fundamental es con la psicología, con la psiquiatría, porque algunos me dicen "yo no necesito, por la Ley" y yo digo “¿Cómo hacemos para volver para atrás de una cirugía de estas? Imposible. Así que tenemos que estar seguros de que vos estas bien para la cirugía. Si te hacemos mujer ¿Cómo volvemos para atrás? Imposible” [...] Si está tratándose ya hace dos o tres años en otro lado, bueno, (pedimos) el certificado del otro psicólogo o psiquiatra que diga que está en condiciones de operarse (R., cirujano).

Si se requerían tratamientos hormonales, la indicación de interconsulta psicoterapéutica dependía del criterio del/a profesional en función de cada caso 
particular. En ambos casos -obligatorio u opcional- el objetivo de la entrevista con un/a profesional de Salud Mental no era el de corroborar un diagnóstico y descartar la psicosis como en los primeros SOC elaborados por la WPATH, sino el de acompañar el proceso de toma de decisión respecto a los tratamientos, y que los/as profesionales/as contaran con garantías respecto a la estabilidad emocional de las personas solicitantes del tratamiento. Tras la aprobación de la Ley de Identidad de Género, para los/as profesionales entrevistados/as la necesidad de garantías no respondía a una responsabilidad legal sino de ética profesional, amparada en la noción de cuidado y salud integral. La atención de la salud basada en estas nociones se encontraba ligada al problema de la gestión de los riesgos tanto somáticos como psicológicos o emocionales asociados a la realización de los tratamientos de construcción corporal. Siguiendo a Michel Foucault (1999), las diferentes formas de gestión de riesgo constituyen estrategias biopolíticas que se vinculan con las mutaciones en las prácticas y discursos médicos como estrategia de gobierno de los cuerpos (FOUCAULT, 1999). Para Mitchell Dean (1998), el riesgo es una forma de ordenar una realidad caótica e imprevisible a fin de tornarla interpretable, medible y proyectable, en definitiva, gobernable.

\section{Del protocolo a la personalización-customización}

Otro de los desplazamientos identificados es el pasaje de un modelo de atención protocolizado compuesto de tres etapas lineales y sucesivas (psicoterapiahormonización-cirugía genital) a una personalización o customización de los tratamientos en función de las necesidades de los y las trans. Para los/as profesionales entrevistados/as, esta modalidad permitía maximizar las probabilidades de "éxito" de las intervenciones en lo que refería a los resultados no solo orgánico-funcionales sino también "simbólicos", término utilizado para referir a la auto representación corporal y el placer sexual. Así lo explicaba uno de los cirujanos urólogos entrevistados:

No es una cirugía nada más. Los cirujanos estamos acostumbrados a pensar en cirugía y nada más, pero si uno se pone a decir que esto es sacar los testículos, sacar el pene, hacer una vagina, no... Tiene un montón de simbolismo. Hay que hablar antes con los pacientes. No todos los pacientes quieren el mismo tipo de cirugía. Hay un tema de customización e individualización de esto, feroz. [...] Nuestras pacientes como cualquier mujer nativa puede tener quilombos sexuales, nuestros pacientes también los tienen: "no me siento, no me encuentro, no llego al orgasmo" cualquier cosa habitual. Bueno, en estos pacientes tiene un simbolismo muchísimo mayor (R., cirujano). 
Los/as profesionales asumían que para ser exitosos/as en este tipo de intervenciones necesitaban desarrollar una sensibilidad que excedía las meras capacidades técnicas: requería de una escucha atenta y comprometida de las necesidades y expectativas de los y las trans. De este modo, no hay protocolo que pueda suplir la sensibilidad necesaria para llevar adelante la tarea, ya que se trata de prácticas que intervienen en un aspecto esencial para la constitución subjetiva de las personas, como lo es la identidad y la sexualidad.

\section{De la minimización del riesgo al cálculo costo-beneficio}

En los protocolos de la WPATH vigentes hasta 2011 el principal riesgo a sopesar era el de arrepentimiento, que en casos extremos podía llevar al suicidio (PRAGIER, 2011). Pero entre 2014 y 2016 la atención era brindada teniendo en cuenta un amplio mapa de riesgos posibles, no solamente el riesgo de arrepentimiento. Se sopesaban una multiplicidad de posibles consecuencias, desde las físicas hasta las de no intervenir la corporalidad. Los/as profesionales partían de la idea de que no existía una situación ideal en la que no hubiera ningún tipo de riesgo. Desde allí discutían con la idea de que un cuerpo ideal era aquel no intervenido ni hormonal ni quirúrgicamente. Desde esta perspectiva, consideraban que tanto la intervención como la no intervención tenían algún tipo de costo o riesgo a sopesar, sea físico o subjetivo. Así lo explicaba un endocrinólogo entrevistado:

Hay mucha conmoción entre los profesionales por el hecho de decir "si vos le vas a dar estrógenos lo estás predisponiendo a la trombosis, si le vas a dar progestágenos lo estás predisponiendo al cáncer de mama y a la osteoporosis" [...]. O sea, piensan generalmente en el ideal, o sea, en su ideal... "Mejor no le doy nada y lo eximo de riesgo" pero se olvidan que si se lo deja en las condiciones en las que está tiene otro riesgo. Entonces hay realidades donde hay que asumir riesgos, porque si vos tenés una persona que no está contenta con su sexo, tenés que elegir entre el riesgo del deterioro de su salud mental o entre el riesgo de una trombosis, no existe la opción del no riesgo (L., endocrinólogo).

Algunos interrogantes que emergen a partir de los desplazamientos discursivos identificados se orientan a cuestionar quién sopesa esos riesgos y quién tiene la última palabra en torno al curso de acción a seguir. La Ley de Identidad de Género legalizó los tratamientos de construcción corporal trans y, por ende, eliminó la potencial denuncia por violación de la Ley de Ejercicio de la Medicina hacia los/as profesionales que los realizaran. Sin embargo, partiendo de la idea de que no hay una situación ideal y que siempre hay riesgos a sopesar, los/as profesionales continuaban 
reclamando su autoridad sobre la definición del curso de los tratamientos, apelando a su responsabilidad de cuidado de índole ético-profesional y a una experiencia y saber clínico insustituible.

Los/as profesionales entrevistados/as realizaban críticas al contenido de la Ley de Identidad de Género y a cualquier legislación que tuviera por objeto regular el ejercicio de su profesión. Criticaban una formulación del concepto de autonomía del paciente que lo entendiera como un individuo consumidor de bienes y servicios biotecnológicos, donde el criterio clínico no tuviera entidad ni razón de ser:

La ley tampoco nos va a decir a nosotros cómo atender al paciente, ni decir cuál es el momento oportuno de indicar tal estudio, o cuál es el momento de darle o no darle la aprobación para operarse, o decirle cuándo no puede realizar tal tratamiento. Todo lo que vos tenés, o todo lo que el médico hace tiene indicaciones, contra indicaciones, efectos adversos, etc. [...] Entonces, hay un montón de cuestiones que se contraindican. Por más que haya una Ley, hay cuestiones médicas. [...] la gente cree que como en el papel dice "a mí me tienen que dar...” y de repente el médico es un quiosquero que se planta ahí y viene el paciente y le dice: "deme esto, deme lo otro" [...] vos como médico no existís y no tenés ningún rol (M., endocrinólogo).

Las demandas del activismo trans local y global frente al campo médico en algunos casos han logrado desestabilizar las certezas de los/as profesionales de salud, y han conseguido promover una revisión crítica de sus propias prácticas. En otros casos, han tenido como respuesta la reafirmación de su autoridad en un sentido defensivo. Las afirmaciones de los/as profesionales de la salud respecto a la obligatoriedad de contar con autorizaciones por parte de profesionales de la Salud Mental para poder acceder a prácticas quirúrgicas contempladas en la Ley de Identidad de Género debieran revisarse, ya que se trata de prácticas que se contradicen con lo establecido en la Ley de Identidad de Género. Vale decir que existe un sinnúmero de prácticas quirúrgicas electivas irreversibles a las que las personas cisgénero se someten regularmente y sobre las cuales el campo médico no establece los mismos preceptos de vigilancia que establece frente a las intervenciones y tratamientos requeridos por las personas trans. Al decir de dichos/as profesionales, estos requisitos se guían por los criterios de cuidado integral de la salud. A fin de adecuar las prácticas de atención al contenido de las legislaciones actualmente vigentes en materia de género y sexualidad, las instituciones de salud debieran avanzar en el diseño de estrategias de cuidado, diálogo y acompańamiento -también referidas por los/as profesionales entrevistados/as para la elaboración de este artículo- que no se reduzcan a la interconsulta obligatoria con 
profesionales de la Salud Mental. Dichas estrategias podrán, a su vez, ser replicadas en toda práctica médica, no exclusivamente en las que impliquen a la atención de la salud de las personas trans.

El proceso de revisión de las prácticas y discursos médicos abierto con la aprobación de la Ley de Identidad de Género invita a indagar en las distintas concepciones en torno a la relación médico/a-paciente sostenidas por profesionales y usuarios/as de salud. Siguiendo a Annemarie Mol (2008), existen dos formas de gestionar la atención en salud que discuten con las formas tradicionales de relación médico/apaciente: la lógica del cuidado y la lógica de la elección. Desde la lógica de la elección, la relación médico/a-paciente adopta la forma de un contrato entre individuos libres, asumiendo los preceptos de la ciudadanía liberal. A su vez, la discusión sobre las potenciales ventajas y riesgos de los tratamientos se reducen a un asunto privado. $\mathrm{La}$ lógica del cuidado, por el contrario, se distancia tanto de la lógica tradicional como de la lógica de la elección. Su puesta en práctica requiere del diálogo y la creatividad profesional. Desde esa perspectiva, tanto los procesos de definición de diagnósticos como las prácticas de atención son resultado del diálogo entre profesionales y usuarios/as de salud. Se trata de procesos flexibles y no universalizables, ya que se definen en cada situación concreta. Bajo la lógica del cuidado, cobran lugar central los saberes de los sujetos sobre sus propios procesos corporales. En definitiva, tiene como horizonte democratizar las formas de relación entre profesionales y usuarios/ as en lo que respecta a los mecanismos de categorización y prácticas de intervención. Sus límites están dados por las posibilidades biotecnológicas disponibles, el estado de los debates morales sobre el cuerpo y la autonomía y la ponderación de los riesgos para la salud (MOL, 2008; ORTEGA ARJONILLA et al., 2014). El desafío entonces es que las tensiones y revisiones suscitadas entre profesionales especializados/as en salud trans a partir de la aprobación de la Ley de Identidad de Género tiendan a afianzar la lógica del cuidado tal como es entendida por Mol.

\section{Reflexiones finales}

En el presente trabajo se analizó la recepción de la Ley de Identidad de Género por parte de profesionales de la salud del Área Metropolitana de Buenos Aires especializados/as en tratamientos hormonales y quirúrgicos de construcción corporal trans. Se identificaron una serie de mutaciones discursivas en los criterios de ingreso 
y evaluación de resultados de dichos tratamientos respecto a los anteriormente vigentes en los manuales diagnósticos y protocolos de atención de circulación global.

En un primer apartado se abordó el nacimiento y desarrollo de los diagnósticos de "Transexualismo" y "Trastorno de la identidad de género", y su incorporación y transformación en los principales manuales diagnósticos de amplia circulación en la comunidad médica local y global. Se expusieron los criterios de ingreso y atención vigentes en los protocolos médicos que desde fines de 1970 sistematizaron normas y criterios de atención para los tratamientos de construcción corporal de las personas trans.

Posteriormente, se analizaron las principales mutaciones discursivas identificadas respecto a los criterios clínicos previamente vigentes en el campo médico: del diagnóstico al acompañamiento, del protocolo a la personalización-customización y de la minimización del riesgo al cálculo costo-beneficio. Dichas mutaciones se dieron en virtud de la aprobación de la Ley de Identidad de Género argentina, que desjudicializa y despatologiza el acceso a los tratamientos médicos de construcción corporal para los y las trans. También se ubican como factores explicativos de dichas mutaciones los cambios en los manuales diagnósticos de circulación global, el avance en la adquisición de derechos por parte las personas LGBT (lesbianas, gays, bisexuales y trans) y la progresiva visibilidad de las demandas de los colectivos trans en el espacio público local.

Las mutaciones discursivas identificadas plantean una serie de interrogantes respecto al modo en que la escucha de las necesidades de los/as pacientes en términos de cuidado y acompańamiento puede plasmarse efectivamente en las prácticas médicas. El reclamo de los/as profesionales de ser reconocidos/as como expertos/ as en su quehacer profesional, y no como meros/as "expendedores/as" de hormonas y cirugías, sitúa el debate en los términos que Annemarie Mol caracterizó como la tensión entre la lógica del cuidado -que requiere del diálogo permanente entre profesionales y pacientes- y lógica de la elección -en la que prima la lógica del contrato entre individuos libres y cada cual se responsabiliza por sus actos y decisiones-. La vigencia de la Ley de Identidad de Género legitima las demandas del activismo de usuarios/as de salud en general, y del activismo trans en particular. No obstante, las transformaciones en las prácticas del campo médico orientadas por la lógica del cuidado requieren de un constante diálogo entre organizaciones, usuarios/as de salud y profesionales en el marco de políticas públicas direccionadas en ese mismo sentido. 


\section{Referencias}

BARBOSA, B. C. Imaginando trans: saberes e ativismos em torno das regulaçóes das transformaçóes corporais do sexo. Tesis (Doctorado) - Faculdade de Filosofia, Letras e Ciências Humanas, Programa de Pós-Graduação em Antropologia Social. Universidade de São Paulo, São Paulo, 2015.

BERKINS, L. Cumbia, copeteo y lágrimas. Informe nacional sobre la situación de las travestis, transexuales y transgéneros, Buenos Aires: ALITT, 2007.

BERKINS, L.; FERNÁNDEZ, J. La gesta del nombre propio: Informe sobre la situación de la comunidad travesti en la Argentina. Buenos Aires: Ed. Madres de Plaza de Mayo, 2004.

CABRAL, M. Post scriptum. In: BERKINS, L. Cumbia, copeteo y lágrimas. Informe nacional sobre la situación de las travestis, transexuales y transgéneros. Buenos Aires: ALITT, 2007. p. $140-146$.

CAULDWELL, D. Psychopathia Transexualis. In: STRYKER, S.; WHITTLE, S. (Eds.) The transgender studies reader. Routledge: New York, 2006. p. 40-44.

COLL-PLANAS, G. La policía del género. In: MISSÉ, M.; COLL-PLANAS, G. (Eds.) El género desordenado. Críticas en torno a la patologización de la transexualidad. Barcelona: Egales, 2010, p. 55-67.

DEAN, M. Risk, Calculable and Incalculable. Soziale Welt, v. 49, p. 25-42, 1998.

DI SEGNI, S. Sexualidades. Tensiones entre la psiquiatría y los colectivos militantes. Buenos Aires: Fondo de Cultura Económica, 2013.

FARJI NEER, A. Fronteras discursivas: travestismo, transexualidad y transgeneridad en los discursos del Estado argentino, desde los Edictos Policiales hasta la Ley de Identidad de Género. Tesis (Maestría) - Maestría en Investigación en Ciencias Sociales, Facultad de Ciencias Sociales, Universidad de Buenos Aires. Buenos Aires, Argentina, 2013.

. Sentidos en disputa sobre los cuerpos trans: los discursos médicos, judiciales, activistas y parlamentarios en Argentina (1966-2015). Tesis (Doctorado) - Doctorado en Ciencias Sociales, Facultad de Ciencias Sociales, Universidad de Buenos Aires, Buenos Aires, Argentina, 2016a.

. Estrategias para garantizar el derecho a la salud integral trans. Un abordaje sociológico de las perspectivas de los profesionales de la salud de la Ciudad Autónoma de Buenos Aires. Revista Argentina de Salud Pública, Ministerio de Salud de la Nación, Argentina, v. 7, n. esp., diciembre, p. 43-44, 2016b.

FOUCAULT, M. ¿Crisis de la medicina o crisis de la antimedicina? In: Estrategias de poder. Barcelona: Paidós, 1999, p. 343-361. 
FRIEDER, K.; ROMERO, M. Ley de identidad de género y acceso al cuidado de la salud de las personas trans en Argentina. Buenos Aires: Fundación Huésped y Asociación de Travestis, transexuales y transgéneros de Argentina, 2014.

HAUSMAN, B. Changing Sex: Transsexualism, Technology, and the Idea of Gender. Durham: Duke University Press, 1995.

MOL, A. The logic of care. Health and the problem of patient choice. London: Routledge, 2008.

ORTEGA ARJONILLA, E. et al. Discurso activista y estatus médico de lo trans: hacia una reconfiguración de cuidados y diagnósticos". In: PÉREZ SEDEÑO, E.; ORTEGA ARJONILlA, E. (Eds.) Cartografías del Cuerpo. Biopolíticas de las ciencias y la tecnología. Valencia: Ediciones Cátedra, 2014. p. 521-567.

PRAGIER, U. M. Trastorno de identidad de género (TIG), un enfoque integral. Revista de la Sociedad Argentina de Endocrinología Ginecológica y Reproductiva, v. 18, n. 2, p. 45-56, 2011.

SUESS, A. Análisis del panorama discursivo alrededor de la despatologización trans: procesos de transformación de los marcos interpretativos en diferentes campos sociales. In: MISSÉ, M.; COLL-PLANAS, G. (Eds.) El género desordenado. Críticas en torno a la patologización de la transexualidad. Barcelona: Egales, 2010. p. 29-54.

\section{Documentos}

Ley $n^{\circ} 26.743$ de Identidad de Género (B. O. 24-05-2012).

Decreto 903/2015 (PEN) Identidad de Género Ley 26.743. Reglamentación Artículo no 11 (B.O. 29-05-2015).

American Psychiatric Association. Diagnostic and Statistical Manual of Mental Disorders, Fifth Edition (DSM-5), 1994.

World Professional Association for Transgender Health (WPATH). Standards of Care (SOC) for the Health of Transsexual, Transgender, and Gender-Nonconforming People, 7th Version, 2011.

\section{Agradecimientos}

Consejo Nacional de Investigaciones Científicas y Técnicas.

Comisión Nacional Salud Investiga - Ministerio de Salud de la República Argentina.

\section{Notas}

${ }^{1}$ Figura establecida en el artículo 270 de la Ley 26.061 de "Protección integral de los derechos de niños, niñas y adolescentes”, cuya misión es la de asegurar las garantías mínimas en los procedimientos judiciales o administrativos. 
${ }^{2}$ Art. $9^{\circ}$ del Decreto $1007 / 2012$.

${ }^{3}$ La primera versión de la Clasificación Internacional de Enfermedades data de 1893. En su $6^{\circ}$ versión del año 1948 incluyó un capítulo sobre trastornos mentales y en su $8^{\circ}$ de 1965 incluyó un apartado de Desviaciones Sexuales incorporando allí las categorías del "Travestismo" y la "Homosexualidad". El CIE 9 de 1978 eliminó la categoría "Homosexualidad" pero incluyó el diagnóstico de "Transexualismo" al interior del apartado de las "Desviaciones sexuales". En el año 1992 se publicó la CIE 10, actualmente vigente. Allí, el "Transexualismo", conjuntamente con el "Travestismo no fetichista” y el "Trastorno de la identidad de género en la infancia", fueron ubicados dentro de la categoría englobadora de "Trastorno de la identidad de género" (TIG) (ubicado en el capítulo dedicado a los "Trastornos mentales y de comportamiento"). El DSM III de 1980 también eliminó la categoría "Homosexualidad” y creó una nueva categoría: el "Trastorno de la identidad sexual". Allí incorporó el diagnostico de "Transexualismo". En la versión del ańo 1994 lo reemplazó por el de "Trastorno de la identidad de Género" que, conjuntamente con las "Parafilias" y las "Disfunciones sexuales" conformaron el apartado de "Trastornos sexuales y de la Identidad de Género” (DI SEGNI, 2013). 


\section{Abstract}

\section{Health professionals facing Gender Identity}

Law. Tensions between expert knowledge and integral care in Argentina

This article addresses the reception of the Gender Identity Law by health professionals from the Metropolitan Area of Buenos Aires. It analyzes a corpus of in-depth interviews with health professionals specialized in gender reaffirmation treatments from the fields of Surgery, Endocrinology and Mental Health. Argentina's Gender Identity Law (26743/12) provides coverage within the Mandatory Medical Plan for surgical procedures and hormonal treatments whose aim is to align body to gender identity. Unlike the previous legal regime, it does not require a diagnostic or judicial authorization to access hormonal or surgical treatments for trans population. The article describes the transformation of discourses of health professionals regarding access to treatment and evaluation of results. The main conclusions establish that the approval of the Gender Identity Law allowed three mutations in discourses prevailing in the medical field: from diagnosis to follow-up; from protocol to customization; from risk minimization to cost-benefit calculation.

> Keywords: gender identity right; health services for transgender people; depathologization; medicalization. 


\section{Resumo}

Profissionais da saúde frente à Lei de Identidade de Gênero argentina. Tensöes entre o conhecimento especializado e o atendimento integral

$\mathrm{O}$ artigo expóe os resultados de uma pesquisa qualitativa baseada em entrevistas em profundidade com profissionais da saúde das especialidades de Saúde Mental, Endocrinologia e Cirurgia, que realizam ou avaliam os ingressos nos tratamentos médicos solicitados pelas pessoas trans. Este artigo pretende trazer algumas reflexóes em torno dos critérios de admissáo e avaliação dos resultados dos tratamentos cirúrgicos e hormonais em pessoas trans, desde a aprovação da Lei de Identidade de Gênero, em 2012. A Lei n. 26.743, de Identidade de Gênero, legalizou as intervençôes médicas para que travestis, transexuais, transgêneros e demais pessoas trans possam construir uma corporalidade conforme sua identidade de gênero. A lei estabeleceu a cobertura dos tratamentos médicos pelo sistema de saúde pública e eliminou o requisito do laudo judicial para o acesso aos procedimentos desejados. Os resultados indicam que a Lei de Identidade de Gênero permitiu três mudanças nos discursos dos profissionais da saúde: do diagnóstico ao acompanhamento; do protocolo à customização; e da minimização dos riscos ao cálculo de custo-benefício.

> Palavras-chave: direito à identidade de gênero; serviços de saúde para pessoas trans; despatologização; medicalização. 